\title{
Numerical Evaluation of Craters Produced by Explosions on the Soil Surface
}

\author{
N.M. NAGY* \\ Military Technical Collage/Civil Eng. Department, Cairo, Egypt
}

\begin{abstract}
Studies of craters produced by explosions above and underneath the ground surface are rarely found in the open technical literature. Most reports are confidential and access is limited to government agencies. Much of the information on explosively formed craters found in the literature is based on experimental data. Numerical studies were very limited until recently. An ablity to predict the anticipated size of crater is crucial to identification of the corresponding damage that might be caused by a given explosive charge, or to assessment of the magnitude of the charge if this is not known. In this paper a non-linear dynamic numerical analysis of the explosion phenomena in clay soils associated with different amounts of TNT explosive charge is performed using the ABAQUS/Explicit computer program. To validate the numerical procedure and material constitutive models used in the present work, a comparison with experimental results is first performed. The results obtained illustrate that the agreement between the numerical and the experimental results is reasonable. A study of the influence of soil density on the crater dimensions is then undertaken. From the numerical results obtained, a new prediction equation is proposed for the crater dimensions as a function of the explosive charge considered. This equation represents the approximation of the numerical results by least squares fitting.
\end{abstract}

DOI: 10.12693/APhysPolA.128.B-260

PACS: 91.30.cd

\section{Introduction}

Following the recent explosive attacks around the world there is a clear need for extensive studies of the explosive effects on soils and crater formations. Investigating and studying the crater formation is a valid means of studying the soil-blast interaction phenomena [1]. Studying the crater formations produced by explosions will give a good means for estimating the explosive charge (mass of explosive) used and give good data about the explosion process. The mechanism of crater formation is complex and is associated with the dynamic physical properties of air, soil and explosive. Even very carefully controlled cratering tests produce deviations in the crater dimensions measured of at least $10 \%$, while differences of as much as $30 \%$ to $40 \%$ are common [2].

The explosive charges used in this paper were chosen to be within the mass range, which might be used in any terrorist attacks against important buildings. These terrorist attacks commonly take place on the ground surface. The range of explosive charge (mass) experienced in such attacks was discussed by Elliot et al. [3] and Ambrosini et al. [4], and also represents the range of the surface mine charges that is normally used in military operations.

In order to validate not only the material models and corresponding properties but also the analysis procedures; a validation stage is initially conducted by comparing the results of a series of physical tests undertaken by Ambrosini et al. [5] with those obtained from detailed numerical modelling of the same physical problem. This

*e-mail: nabilmnagy@yahoocom enables calibration of the model and material parameters. A better understanding of the explosion phenomena is being gradually gained by combining the results from physical experiments and numerical modelling [6, 7]. A numerical investigation of the crater formation obtained using different amounts of charge located on the soil surface is then undertaken. Charges from 20 to $640 \mathrm{~kg}$ are used. In addition a study of the influence of soil properties on the crater dimensions is performed. From the numerical results obtained, an empirical equation is proposed for the prediction of crater diameter resulting from the explosive charges considered. This equation represents the approximation of the numerical results by least squares fitting.

\section{Crater dimensions}

The shape of the crater created by an explosive charge positioned on or below the ground level is shown in cross section in Fig. 1. The crater dimensions are based on the definitions of Kinney and Graham [8] and Cooper [9] which are used in this study. Figure 1 also shows the characteristic features of a crater produced by the explosion in cross-section. The explosion is thus considered to produce four regions of the crater. The first is the plastic zone where the soil has been deformed and compressed to higher than normal density. The second region is the rupture region, which is crushed and damaged because of the explosion. Beyond this is the third region, which is the fallback zone, where the ejected soil returns back to the cavity, hiding the true (original) crater boundary and forming the boundary of the apparent crater. The final region is the ejecta region that forms the outer portion of the crater edge. In Fig. 1, $D$ is the apparent diameter of the crater, $D_{r}$ is the true crater diameter and $d$ is the apparent depth of the crater. 


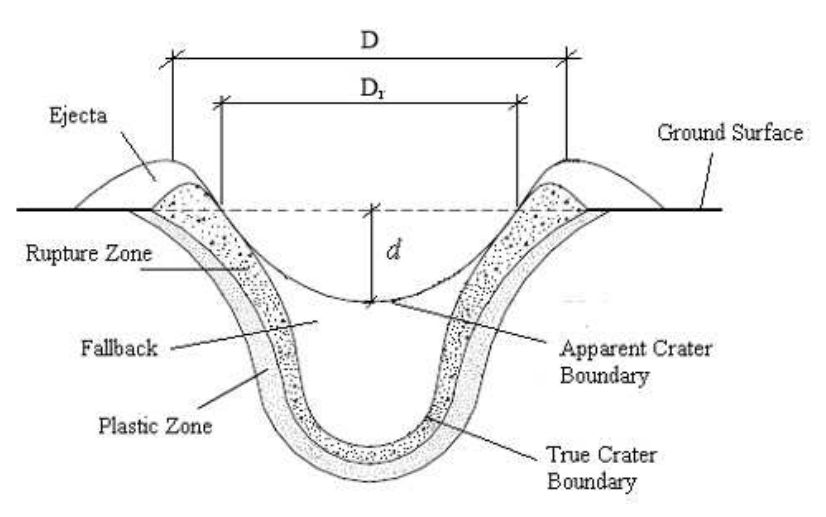

Fig. 1. Cross sectional dimensions of a typical crater.

\section{Finite element model of crater formation}

\subsection{Numerical mesh}

In this paper, the non-linear finite element program ABAQUS/Explicit is used to undertake a twodimensional (2D) axisymmetric analysis of the problem as shown in Fig. 2. The arbitrary Lagrange Euler coupling formulation (ALE) is used in the analysis to eliminate the distortion of the mesh under high deformation. The overall geometric model is divided into three different regions representing the soil, air and explosive materials. A convergence study involving mesh refinement, appropriate selection of element types and simulation of the infinite boundaries was initially performed, whence the optimum meshes were identified for the soil, explosive charge (TNT) and the air, as shown in Fig. 2.



Fig. 2. Mesh for the axisymmetric model.

The soil region is represented by 4-node bilinear axisymmetric quadrilateral, reduced integration, elements (CAX4R). Smaller elements are used in the higher stress region - i.e. near the explosion, with progressively larger elements as the stresses reduce, as shown in Fig. 2. Symmetry boundary conditions are applied along the axis of symmetry by restraining the displacement in the radial direction $(u=0.0)$. The 4 -node, axisymmetric, infinite elements (CINAX4) are used to provide quiet boundaries at the right side and bottom boundaries to the soil mesh. In this manner the ability of the elements to transmit energy out of the finite element mesh without trapping or reflecting it is optimized by simulating a boundary at infinity [10]. In order to obtain a good refinement of the air mesh, and to avoid high distortion errors, the air region is represented by an assemblage of 3-node linear axisymmetric triangular elements (CAX3) in the near field region to the charge. The far field air region is again represented by 4-node bilinear axisymmetric quadrilateral elements (CAX4R), with infinite elements (CINAX4) providing the quiet boundaries at the right hand side and top of the air region. The explosive material zone is meshed by 4-node bilinear axisymmetric quadrilateral, reduced integration, elements (CAX4R). The size of this zone and number of elements in it depend on the magnitude of the explosive charge (i.e. the charge 
mass). The explosive charge is varied from $1 \mathrm{~kg}$ to $640 \mathrm{~kg}$ of TNT, and the mesh dimensions varied accordingly. For small charges in the range 1-20 kg, a mesh of $12 \mathrm{~m} \times 12 \mathrm{~m}$ is used for both the soil and air regions whilst for charges of $40 \mathrm{~kg}$ and more meshs of $30 \mathrm{~m} \times 30 \mathrm{~m}$ is used.

\subsection{Material models}

\subsubsection{Soil model}

The soil is considered as a clay soil whose behaviour is modelled by an elasto-plastic Drucker-Prager Cap model. The Drucker-Prager-Cap model was originally developed to predict the plastic deformation of soils under compression $[11,12]$. It consists principally of two intersecting segments: a shear failure segment $F_{s}$ and a cap segment $F_{c}$ which provides an inelastic hardening mechanism to account for plastic compaction and helps to control volume dilatancy when the material yields in shear. A transition segment Ft has been introduced to provide a smooth surface between the shear failure surface and cap segment. Figure 3 shows the segments in the $p-q$ plane where $p$ is the equivalent pressure stress and $q$ is the Misses equivalent stress.

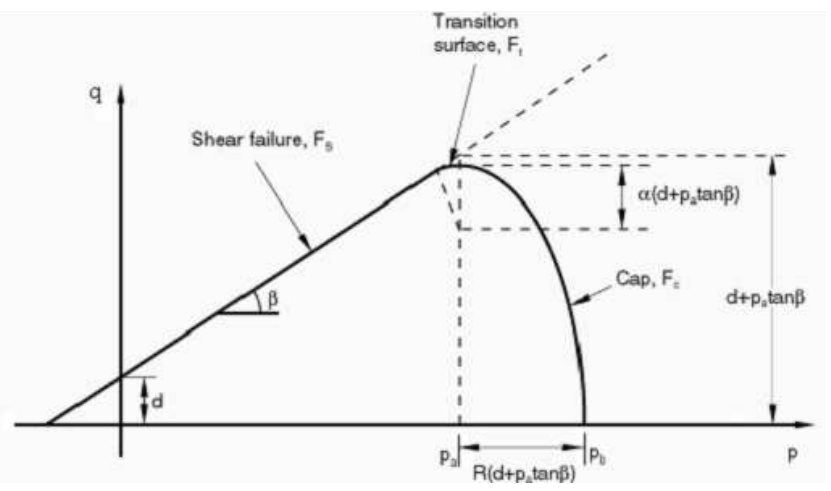

Fig. 3. The Drucker-Prager cap model [10].

The two stress invariants $p$ and $q$ can be defined as:

$$
\begin{aligned}
& p=\frac{1}{3}\left(\sigma_{1}+\sigma_{2}+\sigma_{3}\right), \\
& q=\sqrt{\frac{1}{6}\left[\left(\sigma_{1}-\sigma_{2}\right)^{2}+\left(\sigma_{2}-\sigma_{3}\right)^{2}+\left(\sigma_{1}-\sigma_{3}\right)^{2}\right]},
\end{aligned}
$$

where $\sigma_{1}, \sigma_{2}$ and $\sigma_{3}$ are the principal stresses. The shear failure segment in the Drucker-Prager cap model provides a criterion for the occurrence of shear flow, which is dependent upon the cohesion $d$ and the angle of internal friction $\beta$ of particulate materials according to the Mohr-Coulomb hypothesis:

$$
F_{s}=q-p \tan \beta-d=0 .
$$

The cap segment, which intersects the equivalent pressure stress axis, is an elliptical curve with constant eccentricity in the $p-q$ plane as given by:

$$
\begin{gathered}
F_{c}=\sqrt{\left(p-p_{a}\right)^{2}+\left(\frac{R_{t}}{1+\alpha-\alpha \cos \beta}\right)^{2}} \\
-R\left(d+p_{a} \tan \beta\right)=0,
\end{gathered}
$$

where $R$ and $\alpha$ are parameters determining the shape of the cap segment and the smooth transition surface between the shear failure segment and the cap segment, respectively. $p_{a}$ is an evolution parameter representing the hardening or softening driven by the volumetric plastic strain, and is given by:

$$
p_{a}=\frac{p_{b}-R d}{1+R \tan \beta}
$$

where $p_{b}$ is the hydrostatic pressure yield surface that defines the position of the cap. $p_{b}$ is generally assumed to be dependent upon the volumetric inelastic strain $\varepsilon_{v o l}^{i n}$. This dependence controls the hardening or softening of the cap segment; volumetric plastic compaction results in hardening, while volumetric plastic dilation develops softening. In order to ensure that the primary feature of the Drucker-Prager Cap model is not significantly modified by the introduction of the transition segment, for the sake of numerical implementation the transition segment is always kept relatively small by restricting the parameter $\alpha$ to typical values of 0.01 to 0.05 . The transition segment is given by:

$$
\begin{aligned}
& F_{c}=\sqrt{\left(p-p_{a}\right)^{2}+\left[q-\left(1-\frac{\alpha}{\cos \beta}\right)\left(d+p_{a} \tan \beta\right)\right]^{2}} \\
& -\alpha\left(d+p_{a} \tan \beta\right)=0 .
\end{aligned}
$$

Full calibration of the model described above requires triaxial test data. In the numerical analysis of this paper, the soil properties included are obtained from the actual properties of the clay soil used in the physical tests presented by Ambrosini et al. [5]. However, these properties are not sufficient to fully specify the parameters required to define a Drucker-Prager Cap model. Therefore, the parameters required to define the cap plasticity model are determined by a best match between the material properties obtained experimentally by Ambrosini et al. [5] and those quoted in the ABAQUS Example Manual [13]. The soil properties and the corresponding cap plasticity parameters used are given in Table I.

TABLE I

Material properties of the clay soil.

\begin{tabular}{l|c}
\hline \hline Parameter & Value \\
\hline Young modulus $(E)$ & $494 \mathrm{MPa}$ \\
\hline Poisson ratio $(\nu)$ & 0.17 \\
\hline Density $(\rho)$ & $1920 \mathrm{~kg} / \mathrm{m}^{3}$ \\
\hline Material cohesion $(d)$ & $1.38 \mathrm{MPa}$ \\
\hline Material angle of friction $(\beta)$ & $40.4^{\circ}$ \\
\hline Cap eccentricity parameter $(R)$ & 0.3 \\
\hline Initial cap yield surface position $\left(\varepsilon_{v}\right)$ & 0.02 \\
\hline Transition surface radius parameter $(\alpha)$ & 0.01 \\
\hline & $2.75 \mathrm{MPa}, 0.00$ \\
$\quad$ Cap hardening behaviour & $4.83 \mathrm{MPa}, 0.02$ \\
(stress, plastic volumetric strain) & $5.15 \mathrm{MPa}, 0.04$ \\
& $62.0 \mathrm{MPa}, 0.08$
\end{tabular}




\subsubsection{Air model}

The air is modelled using the ideal gas equation of state. This is one of the simplest forms of equation of state for gases [14] which is implemented in ABAQUS and can be written in the form:

$$
P+P_{A}=\rho R\left(\theta-\theta^{Z}\right),
$$

where $P_{A}$ is the ambient pressure, $R$ is the gas constant, $\theta$ is the current temperature, and $\theta^{Z}$ is the absolute zero on the temperature scale being used. In general, the value $R$ for any gas can be estimated as a function of state (e.g., pressure or temperature). The ideal gas approximation is adequate in any region where this value is constant. At constant volume the specific heat $c_{v}$ should be specified for an ideal gas. $c_{v}$ is related to the specific heat at constant pressure, $c_{p}$, by the following equation:

$$
R=c_{p}-c_{v} .
$$

In all analyses the air around the explosion area has been modelled as an ideal gas with the following properties presented by Batchelor [15]. Table II presents the air model properties.

\section{TABLE II}

Material properties of air

\begin{tabular}{l|c}
\hline \hline Parameter & Value \\
\hline Gas constant, $R$ & $287.1 \mathrm{~J} / \mathrm{kg} / \mathrm{K}$ \\
Ambient pressure $P_{A}$ & $101.36 \mathrm{KPa}$ \\
Specific heat & $717.98 \mathrm{~J} / \mathrm{kg} / \mathrm{K}$ \\
Reference density & $1.225 \mathrm{~kg} / \mathrm{m}^{3}$ \\
Reference temperature & $15^{\circ} \mathrm{C}$
\end{tabular}

In view of the fact that the air is a gaseous material and has no ability to support either shear stresses or negative pressures, no strength or failure relations are mandatory for this material [16].

\subsubsection{Charge model}

The charge is modelled using the Jones-Wilkens-Lee (JWL) equation of state. This simulates the pressure generated by expansion of the detonation product and chemical energy of a chemical explosive $[17,18]$. This model has been widely used in engineering applications. The JWL equation of state can be written in terms of the initial energy per unit mass, $E_{m 0}$ as follows [19]:

$$
\begin{aligned}
P & =A\left(1-\frac{\omega \rho}{R_{1} \rho_{0}}\right) \exp \left(-R_{1} \frac{\rho_{0}}{\rho}\right) \\
& +B\left(1-\frac{\omega \rho}{R_{2} \rho_{0}}\right) \exp \left(-R_{2} \frac{\rho_{0}}{\rho}\right)+\frac{\omega \rho^{2}}{\rho_{0}} E_{m 0},
\end{aligned}
$$

where $A, B, R_{1}, R_{2}$ and $\omega$ are material constants which for many common explosives have been determined from dynamic experiments. $\rho_{0}$ is the density of the explosive and $\rho$ is the density of the detonation products. The initial relative density $\left(\rho / \rho_{0}\right)$ used in the JWL equation is assumed to be unity, therefore nonzero values of initial specific energy $E_{m 0}$ should be specified. In the analyses the TNT explosive charges have been modelled by The JWL equation of state with properties as presented by Zhongqi et al. [20] and in the ABAQUS Example Manual [13]. The parameters for the TNT are listed in Table III.

TABLE III

JWL parameters used for modelling TNT explosive.

\begin{tabular}{l|c}
\hline \hline Parameters & Value \\
\hline Detonation wave speed & $6930 \mathrm{~m} / \mathrm{s}$ \\
$A$ & $373.8 \mathrm{GPa}$ \\
$B$ & $3.747 \mathrm{GPa}$ \\
$R_{1}$ & 4.15 \\
$R_{2}$ & 0.9 \\
$\omega$ & 0.35 \\
Explosive density, $\rho_{0}$ & $1630 \mathrm{~kg} / \mathrm{m}^{3}$ \\
Initial specific energy $E_{m 0}$ & $3.63 \mathrm{~J} / \mathrm{kg}$
\end{tabular}

\section{Comparison between numerical and experimental results}

Numerical formation of the whole crater for the case of $10 \mathrm{~kg}$ of TNT located on the soil surface is shown in Fig. 4 at different times after the explosion. The figure also shows the Von Misses stresses developed in the soil and the crater progression from $4 \mathrm{~ms}$ to $12 \mathrm{~ms}$. It can be seen that immediately after detonation the value of stress is high in the region close to the explosion-soil interface. With time, the stress wave propagates into the soil mass resulting in an attenuation of the stresses. This results in the formation of rupture and plastic zones as described in Fig. 1. Also shown in Fig. 4 is the ejecta whose height increases with time after explosion. The maximum height of the ejecta is reached at $12 \mathrm{~ms}$ after the explosion. The results of crater formation after $12 \mathrm{~ms}$ provides no additional information, which indicates that $12 \mathrm{~ms}$ is sufficient for the completion of crater formation.

In order to verify the finite element analysis, a comparison with experimental results is first carried out. The results of a series of tests performed by [5] with different amounts of explosive from $1 \mathrm{~kg}$ to $10 \mathrm{~kg}$ on the soil surface are used to calibrate the material and model parameters. The explosive charges used in these tests were Gelamon 80 a NG (Nitro Glycerine) based gelatinous explosive theoretically equivalent in mass to $80 \%$ of TNT. In order to perform a validation and comparative analysis the mass of explosive was defined by TNT equivalent mass. In order to get the corresponding mass for any other kinds of explosive, the concept of TNT equivalence stated by Formby and Wharton [21] and Smith and Hetherington [22] can be used. The results of the validation exercise are presented in Table IV.

The mean value of the numerical results is $92.7 \%$ of the equivalent experimental results. It can be seen therefore that there is good agreement between the numerical results and the corresponding experimental ones, which enables us to be confident not only in the material models and material properties but also in the mesh design and 

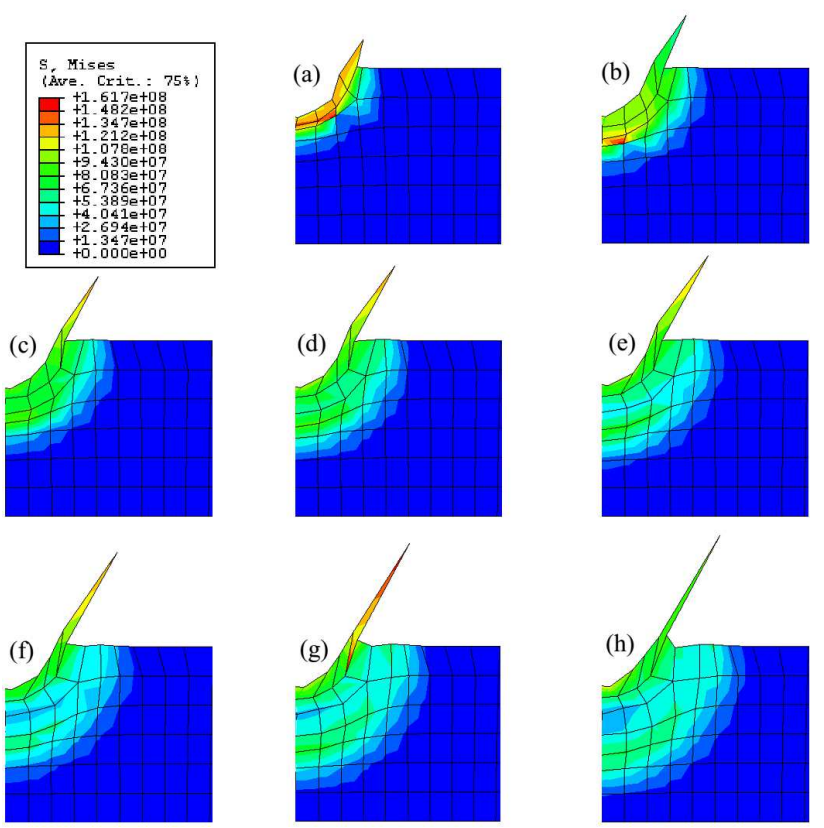

Fig. 4. Crater formation of surface $10 \mathrm{~kg}$ TNT explosion at: (a) $t=4 \mathrm{~ms}$, (b) $t=6 \mathrm{~ms}$, (c) $t=7 \mathrm{~ms}$, (d) $t=8 \mathrm{~ms}$, (e) $t=9 \mathrm{~ms}$, (f) $t=10 \mathrm{~ms}$, (g) $t=11 \mathrm{~ms}$, (h) $t=12 \mathrm{~ms}$.

TABLE IV

Numerical results for crater diameter and comparison with the equivalent experimental results.

\begin{tabular}{c|c|c|c|c}
\hline \hline Case no. & $M[\mathrm{~kg}]$ & $\begin{array}{c}D_{r}[\mathrm{~m}] \\
\text { exp. [5] }\end{array}$ & $\begin{array}{c}D_{r}[\mathrm{~m}] \\
\text { calc. }\end{array}$ & $\begin{array}{c}\text { calc. } / \text { exp. } \\
{[\%]}\end{array}$ \\
\hline 1 & 1 & 0.58 & 0.502 & $86.6 \%$ \\
2 & 2 & 0.74 & 0.72 & $97.3 \%$ \\
3 & 4 & 0.84 & 0.88 & $104 . \%$ \\
4 & 7 & 1.48 & 1.226 & $82.8 \%$ \\
5 & 10 & 1.56 & 1.45 & $92.9 \%$
\end{tabular}

analysis procedures. These results are presented graphically in Fig. 5 with the crater diameter plotted as a function of the cubic root of the explosive mass $M$. It can again be seen that there is a good agreement between the numerical and experimental results. The numerical results are also used to obtain a best-fit line passing through the origin which can be represented by the following equation:

$$
D_{r}=0.6172 M^{1 / 3} \text {. }
$$

The $R$-square value for this line is 0.968 . From Fig. 5 . it can be seen that the line is also close to the experimental results again confirming good agreement between numerical and experimental results. Kinney and Graham [8] undertook a statistical study of two hundred surface explosion tests to obtain an equation for crater diameter $D$, in metres, in terms of explosive mass $M$, in kilograms TNT. Their equation is stated as follows.

$$
D_{r}=0.8 M^{1 / 3} \text {, }
$$

where; $D$ is the crater diameter in metres and $M$ is the explosive mass in kilograms TNT. It can be seen from Fig. 5 that this equation does not correlate with the physically obtained results of Ambrosini et al [5] as well as the numerical results obtained here.

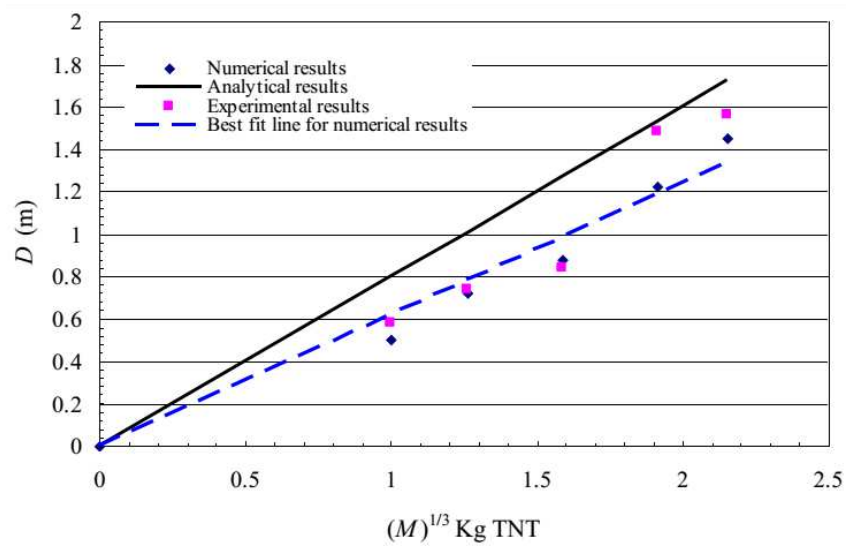

Fig. 5. Crater diameters produced by explosions.

\section{Numerical simulations of large explosive charges}

The laboratory tests reported by Ambrosini et al. [5] inevitably used only small explosive charges. Accordingly further numerical solutions have been established for the crater dimensions created from the TNT charges from $20 \mathrm{~kg}$ to $640 \mathrm{~kg}$ placed directly on the ground surface to simulate more closely the situations of practical interest discussed in the Introduction. All the material models, material properties and the analysis procedures for these larger charge masses are the same as those defined for the small explosive charges considered above except that the soil mesh area is taken as $30 \mathrm{~m} \times 30 \mathrm{~m}$. The numerical results for the crater diameter as a result of using large quantities of explosive charge are presented in Table V.

TABLE V

The numerical results for the crater diameter formed from large surface explosions.

\begin{tabular}{c|c}
\hline \hline$M[\mathrm{~kg}]$ & $D_{r}[\mathrm{~m}]$ \\
\hline 20 & 1.84 \\
40 & 1.96 \\
80 & 2.23 \\
160 & 2.79 \\
320 & 3.14 \\
640 & 5.17
\end{tabular}

These results in conjunction with the numerical results for small charges are presented graphically in Fig. 6 with the crater diameter again plotted as a function of the cubic root of the explosive charge mass $M$. In this figure the best-fit line is also shown for the whole range of explosive charges and its equation is given by: 


$$
D_{r}=0.55679 M^{1 / 3} \text {. }
$$

Equation (12) is considered preferable to Eq. (10), since it is obtained over a much wider range of explosive charges the difference between the two at small charges is relatively small. Equation (12) can therefore be recommended for prediction of the crater diameter formed from surface explosions in soils similar to that used in its derivation. In order to increase the range of applicability of Eq. (12), its applicability for prediction of crater diameter in clay soils with different densities is now investigated.

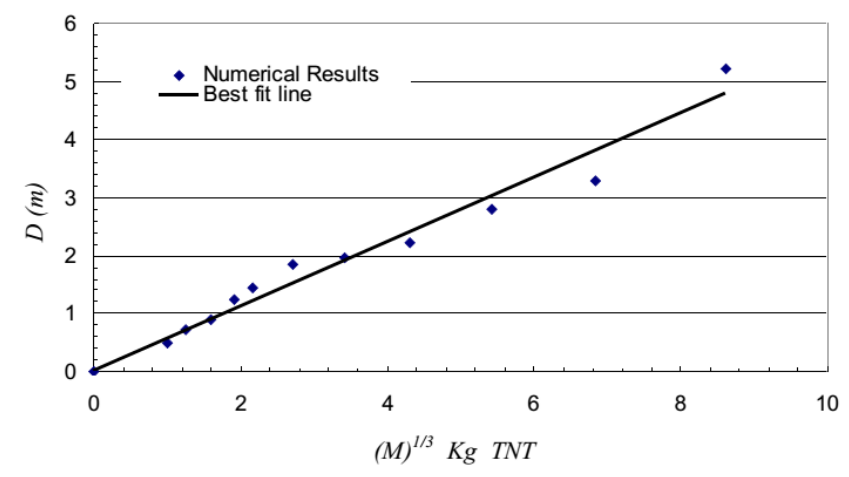

Fig. 6. Numerical results of crater diameter for surface explosions.

\section{Influence of soil properties on soil explosion effects}

Ambrosini et al. [4] investigated the effect of the elastic soil properties on the crater diameter resulting from surface explosions and it was found that variation of the Shear Modulus does not affect the dimensions of the crater significantly. Variation in crater development after the surface explosion gives an indication of the importance of representative changes in soil properties. Accordingly additional numerical investigations are being carried out by varying some of the soil properties. The effect of soil mass density has been investigated using the overall numerical model described above by considering a range of soil densities $\rho_{s}$ from $1300 \mathrm{~kg} / \mathrm{m}^{3}$ to $2000 \mathrm{~kg} / \mathrm{m}^{3}$. The results obtained are presented in Table VI:

Table VI demonstrates that the mass density variation of the soil does not influence significantly the results for the crater diameters. However, it will be necessary to investigate variation of all the soil parameters before more generalized conclusions can be made. Therefore, Eq. (12) can be used for prediction of the crater diameter in clay soils independent of density, but with properties otherwise as defined in Table I.

\section{Conclusions}

This paper presents a fully coupled numerical analysis of air-soil-explosion interaction. The analysis procedures
The effect of soil density on crater diameter.

\begin{tabular}{c|c|c|c}
\hline \hline \multirow{2}{*}{$M[\mathrm{~kg}]$} & \multicolumn{2}{|c|}{$D_{r}[\mathrm{~m}]$} & $D_{1} / D_{2} \%$ \\
\cline { 2 - 3 } & $\rho_{s}=1300 \mathrm{~kg} / \mathrm{m}^{3}$ & $\rho_{s}=1920 \mathrm{~kg} / \mathrm{m}^{3}$ & \\
\hline 20 & 1.92 & 1.84 & $104.34 \%$ \\
40 & 2.10 & 1.96 & $107.14 \%$ \\
80 & 2.32 & 2.23 & $104.04 \%$ \\
160 & 2.82 & 2.79 & $101.1 \%$ \\
320 & 3.33 & 3.14 & $106.1 \%$ \\
640 & 5.22 & 5.17 & $101 \%$
\end{tabular}

and the material models in the fully coupled analysis are validated by comparison with physical tests. Good agreement is obtained between them. Further numerical studies have been performed using the fully coupled model to simulate the effects of large explosive charges. Based on the full set of numerical results, a new equation is presented for the prediction of crater diameter produced by explosions in clay soils taking the variation of soil density into account. This demonstrates that a simple linear equation can be used to predict the crater dimensions as a function of the explosive charge mass. Further studies are underway to investigate the crater formation in different soils considering parametric variation of all soil properties.

\section{References}

[1] P.A. Persson, R. Holmberg, J. Lee, Rock blasting and explosives engineering, CRC Press, USA 1994.

[2] J.W. Bull, C.H. Woodford, Comp. Struct. 69, 695 (1998).

[3] C.L. Elliot, G.C. Mays, P.D. Smith, Proceeding of Institution of Civil Engineers, Structures and Buildings, Vol. 94, Issue 3, 1992.

[4] D. Ambrosini, B. Luccioni, R. Danesi, Mecán. Comput. XXIII, (2004).

[5] R.D. Ambrosini, B.M. Luccioni, R.F. J.D. Danesi, Riera, M.M. Rocha, Shock Waves 12, 69 (2002).

[6] M.P. Braid, D. Bergeron, Experimental Investigation and Analysis of the Effects of Anti-Personnel Landmine Blasts, Defense Suffield Publication, Canada 2001, p. 188.

[7] G. Fairlie, D. Bergeron, 17th Military Aspects of Blast Symposium, Las Vegas 2002.

[8] G.F. Kinney, K.J. Graham, Explosive shocks in air, Springer, New York 1985.

[9] P.W. Cooper, Explosives Engineering, Wiley-VCH, 1997.

[10] ABAQUS Theory Manual, version 6.4, Hibbitt, Karlsson, Sorensen, 2003.

[11] D.C. Drucker, W. Prager Quart. Appl. Math. 10, 157 (1952).

[12] E. Mizuno, W.F. Chen, Nonlinear Analysis in Soil Mechanics Theory and Implementation, Elsevier Science, Amsterdam 1990.

[13] ABAQUS Example Problems Manual, version 6.4, Hibbitt, Karlsson, Sorensen, 2003. 
[14] B.K. Hodge, K. Koenig, Compressible Fluid Dynamics, Prentice Hall, Englewood Cliffs 1995.

[15] G.K. Batchelor, An Introduction to Fluid Dynamics, Cambridge Un. Press, Cambridge 2000.

[16] M. Grujicic, B. Pandurangan, B.A. Cheeseman, Multidisc. Model. Mater. Struct. 2, 363 (2006).

[17] E.L. Lee, H.C. Hornig, J.W. Kury, Adiabatic Expansion of High Explosive Detonation Products, Lawrence Radiation Laboratory, University of California, UCRL-50422, Berkeley 1968.

[18] E. Lee, M. Finger, W. Collins, JWL equations of state coefficient for high explosives, Lawrence Livermore Laboratory, Livermore, Calif, UCID-16189, Berkeley 1973.
[19] ABAQUS Analysis User's Manual, version 6.4, Hibbitt, Karlsson, Sorensen, 2003.

[20] W. Zhongqi, H. Hong, L. Yong, Int. J. Num. Analyt. Meth. Geomech. 28, 33 (2004).

[21] S. Formby, R.K. Wharton, J. Hazard. Mater. 50, 183 (1996).

[22] P.D. Smith, J.G. Hetherington, Blast and Ballistic Loading of Structures, Butterworth Heinemann, Oxford 1994. 